\title{
Pinning Synchronization for Complex Networks with Interval Coupling Delay by Variable Subintervals Method and Finsler's Lemma
}

\author{
Dawei Gong, ${ }^{1}$ Frank L. Lewis, ${ }^{2,3}$ Liping Wang, ${ }^{4}$ Dong Dai, ${ }^{5}$ and Shuang Zhang ${ }^{6}$ \\ ${ }^{1}$ School of Mechatronics Engineering, University of Electronic Science and Technology of China, Chengdu 611731, China \\ ${ }^{2}$ UTA Research Institute, The University of Texas at Arlington, Arlington, TX 76118, USA \\ ${ }^{3}$ Northeastern University, Shenyang 110036, China \\ ${ }^{4}$ Department of Mechanical Engineering, Tsinghua University, Beijing 100084, China \\ ${ }^{5}$ School of Electric Power, South China University of Technology, Guangzhou 510641, China \\ ${ }^{6}$ School of Astronautics and Aeronautic, University of Electronic Science and Technology of China, Chengdu 611731, China
}

Correspondence should be addressed to Dawei Gong; pzhzhx@126.com

Received 18 March 2017; Accepted 4 May 2017; Published 8 June 2017

Academic Editor: Junpei Zhong

Copyright (C) 2017 Dawei Gong et al. This is an open access article distributed under the Creative Commons Attribution License, which permits unrestricted use, distribution, and reproduction in any medium, provided the original work is properly cited.

\begin{abstract}
The pinning synchronous problem for complex networks with interval delays is studied in this paper. First, by using an inequality which is introduced from Newton-Leibniz formula, a new synchronization criterion is derived. Second, combining Finsler's Lemma with homogenous matrix, convergent linear matrix inequality (LMI) relaxations for synchronization analysis are proposed with matrix-valued coefficients. Third, a new variable subintervals method is applied to expand the obtained results. Different from previous results, the interval delays are divided into some subdelays, which can introduce more free weighting matrices. Fourth, the results are shown as LMI, which can be easily analyzed or tested. Finally, the stability of the networks is proved via Lyapunov's stability theorem, and the simulation of the trajectory claims the practicality of the proposed pinning control.
\end{abstract}

\section{Introduction}

Complex networks have large size and nontrivial complex topological features have been intensively studied by many researchers in recent years. Such networks have connections which are neither purely regular nor purely random. These networks are used to understand and predict the behavior of many structures, for example, Internet, medicine, society, and biology.

It has been found that lots of phenomena in real world can be studied by complex networks (such as [1-5] and references therein). Amongst all the topics which are studied by complex networks, synchronization phenomena play an important role due to their real world potential applications. There are many interesting synchronization phenomena in nature world. Lots of efforts have been put into the development of the synchronization problems in complex networks [6-11].
It should be noticed that time-varying delays occur commonly in connection topology of networks which are more realistic and cover more situations in practice. Therefore, various kinds of delay methods have been proposed, and synchronization problems for networks with delay have been extensively studied [12-14]. However, the methods to deal with the delay in these papers always need large amount of calculation. So how to remove the redundant computation and improve networks' performance is still a challenging objective.

Normally, complex networks cannot synchronize by themselves, and some controllers are designed to force the system to be synchronized. However, it is hard to design or realize controllers for all nodes of large network structure. Therefore, pinning controllers have been widely used to synchronize complex networks. In [15], an adaptive predictive pinning control is proposed to suppress the cascade in 
coupled map lattices (CMLs). In [16], by using piecewise Lyapunov theory, some less conservative criteria are deduced for exponential synchronization of the complex networks. In [17], a new adaptive intermittent scheme is used to deduce some novel criteria by utilizing a piecewise auxiliary and other relative references [18-21]. However, in the above papers, many useful situations such as some novel delay processing methods and Finsler's Lemma which can introduce more matrix-valued coefficients to synchronization criteria are not utilized. As far as I know, such pinning synchronization methodology for complex networks has not been proposed yet.

Motivated by the former discussions, we elaborate pinning synchronization results for complex networks via subintervals delay method and Finsler's Lemma. By constructing a novel Lyapunov-Krasovskii function (LKF) and using some mathematical skills proposed in this paper, complex networks can achieve synchronization.

\section{Notations}

$R^{n} \leftrightarrow n$-dimensional Euclidean space

$R^{m \times n} \leftrightarrow m \times n$ real matrices

$I_{n} \leftrightarrow n$-dimensional identity matrix

$A \otimes B \leftrightarrow$ Kronecker product of matrices $A$ and $B$

$\operatorname{diag}(\cdots) \leftrightarrow$ block-diagonal matrix

$\left[\begin{array}{ll}X & Y \\ * & Z\end{array}\right] \leftrightarrow\left[\begin{array}{cc}X & Y \\ Y^{T} & Z\end{array}\right]$

\section{Preliminaries}

Consider the system which consists of $N$ nodes, and each node has an $n$-dimensional subsystem; then the pinning control system can be written as

$$
\begin{gathered}
\dot{x}_{i}(t)=f\left(x_{i}(t)\right)+c \sum_{j=1}^{N} g_{i j} A x_{j}(t-\tau(t))+u_{i}, \\
\quad i=1,2, \ldots, l, \\
\dot{x}_{i}(t)=f\left(x_{i}(t)\right)+c \sum_{j=1}^{N} g_{i j} A x_{j}(t-\tau(t)), \\
i=l+1, l+2, \ldots, N,
\end{gathered}
$$

$x_{i}(t)=\left(x_{i 1}(t), x_{i 2}(t), \ldots, x_{i n}(t)\right)^{T} \in R^{n} \cdot f\left(x_{i}(t)\right) \in R^{n}$ is the activation function. The constants $c(c>0), A=$ $\left(a_{i j}\right)_{n \times n}$, and $G=\left(g_{i j}\right)_{N \times N}$ are, respectively, representing coupling strength, inner-coupling matrix, and outer-coupling connections.

$\tau(t)$ satisfies

$$
\begin{gathered}
0 \leq \tau(t) \leq \tau, \\
\dot{\tau}(t) \leq \mu<1 .
\end{gathered}
$$

$u_{i}, i=1,2, \ldots, N$, are the pinning controllers, which are designed as

$$
u_{i}(t)=-c \eta_{i} A\left(x_{i}(t-\tau(t))-s(t-\tau(t))\right) \in R^{n} .
$$

Let $\eta_{i}>0$, for $i=1,2, \ldots, l$, and $\eta_{i}=0$, for $i=l+1, l+$ $2, \ldots, N, \eta_{i}$ are the control gains. Then we can get

$$
\begin{aligned}
\dot{x}_{i}(t)= & f\left(x_{i}(t)\right)-f(s(t)) \\
& +c \sum_{j=1}^{N} g_{i j} A\left(x_{j}(t-\tau(t))-s(t-\tau(t))\right) \\
& -c \eta_{i} A\left(x_{i}\left(t-\tau_{i}\right)-s\left(t-\tau_{i}\right)\right), \\
& i=1,2, \ldots, N .
\end{aligned}
$$

In this following, we will introduce some elementary situations.

Assumption 1. The outer-coupling matric $G$ satisfies

$$
\begin{aligned}
& g_{i j}=g_{j i} \geq 0, \quad i \neq j \\
& g_{i i}=-\sum_{j=1, j \neq i}^{N} g_{i j}, \quad i, j=1,2, \ldots, N .
\end{aligned}
$$

Definition 2. System (2) is synchronized if

$$
x_{1}(t)=\cdots=x_{N}(t)=s(t), \quad t \longrightarrow \infty,
$$

where $\dot{s}(t)=f(s(t))$ and $s(t)$ is a solution of an isolate node.

Lemma 3 (see [22]). The eigenvalues of $G$ in system (2) is defined by

$$
\lambda_{N} \leq \lambda_{N-1} \leq \cdots \leq \lambda_{3} \leq \lambda_{2} \leq \lambda_{1}=0
$$

On the other hand, if $N-1$ of $n$-dimensional differential equations of their 0 solution are asymptotically stable

$$
\dot{w}_{k}(t)=c \lambda_{k} A w_{k}(t-\tau(t))+J(t) w_{k}(t) .
$$

The Jacobian matrix of $f(x(t))$ at $s(t)$ is $J(t)$; then the synchronized states (2) are the same as the asymptotically stable results of system (9).

Lemma 4 (Jensen's inequality). For positive definite symmetric matrices $\omega \in R^{n \times n}, \omega:[0, \rho] \rightarrow R^{n}$, scalar $\rho>0$, we have

$$
\begin{aligned}
& \rho \int_{0}^{\rho} \omega^{T}(s) \Upsilon \omega(s) d s \\
& \quad \geq\left(\int_{0}^{\rho} \omega(s) d s\right)^{T} \Upsilon\left(\int_{0}^{\rho} \omega(s) d s\right) .
\end{aligned}
$$

Lemma 5 (Finsler's Lemma). Let $\varsigma \in R^{n}, \phi^{T}=\phi \in R^{n \times n}$, and $\mathfrak{B} \in R^{m \times n}, \operatorname{rank}(\mathfrak{B})<n$. Then

(1) $\varsigma^{T} \phi \varsigma<0, \mathfrak{B} \varsigma=0, \varsigma \neq 0$

(2) $\left(\mathfrak{B}^{\perp}\right)^{T} \phi \mathfrak{B}^{\perp}<0$,

(3) $\exists L \in R^{n \times m}, \Phi+L \mathfrak{B}+\mathfrak{B}^{T} L^{T}<0$,

where $\mathfrak{B}^{\perp}$ is a right orthogonal complement of $\mathfrak{B}$. 
Lemma 6 (see [23]). Let $x(t) \in R^{n}, M_{1}, M_{2} \in R^{n \times n}, \chi^{T}=$ $\chi>0 \in R^{n \times n}, Z \in R^{2 n \times 2 n}$, a constant $\tau \geq 0$, and then the integral inequality is defined as follows:

$$
-\int_{t-\tau}^{t} \dot{x}^{T}(s) \chi \dot{x}(s) d s \leq \xi^{T}(t) \Upsilon \xi(t)+\tau \xi^{T}(t) Z \xi(t),
$$

where

$$
\begin{aligned}
\Upsilon & :=\left[\begin{array}{cc}
M_{1}^{T}+M_{1} & -M_{1}^{T}+M_{2} \\
* & -M_{2}^{T}-M_{2}
\end{array}\right], \\
\xi(t) & :=\left[\begin{array}{c}
x(t) \\
x(t-\tau)
\end{array}\right], \\
Z & =\left[\begin{array}{c}
M_{1}^{T} \\
M_{2}^{T}
\end{array}\right] \chi^{-1}\left[\begin{array}{ll}
M_{1} & M_{2}
\end{array}\right] .
\end{aligned}
$$

\section{Main Results}

Theorem 7. For positive definite symmetric matrices $P_{k}, Q_{k}$, and $S_{k}$, real matrices $M_{i k},(i=1,2)$, and the following LMIs hold for all $2 \leq k \leq N$ :

$$
\Xi_{1 k}=\left[\begin{array}{ccc}
H_{1 k} & \tau \Gamma_{1}^{T} S_{k} & \tau(1-\mu) \Gamma_{2}^{T} \\
* & -\tau S_{k} & 0 \\
* & * & -\tau(1-\mu) S_{k}
\end{array}\right]<0,
$$

where

$$
\begin{aligned}
\Gamma_{1} & =\left[J(t), c \lambda_{k} A\right], \\
\Gamma_{2} & =\left[M_{1 k}, M_{2 k}\right], \\
H_{1 k} & =\left[\begin{array}{cc}
\Omega_{11} & \Omega_{12} \\
* & \Omega_{22}
\end{array}\right] . \\
\Omega_{11} & =P_{k} J(t)+J(t)^{T} P_{k}+Q_{k}+(1-\mu)\left(M_{1 k}^{T}+M_{1 k}\right) \\
\Omega_{12} & =P_{k} c \lambda_{k} A-(1-\mu)\left(M_{1 k}^{T}-M_{2 k}\right) \\
\Omega_{22} & =-(1-\mu) Q_{k}-(1-\mu)\left(M_{2 k}^{T}+M_{2 k}\right) .
\end{aligned}
$$

Then system (2) is synchronized.

Proof. The Lyapunov function is confined in the following:

$$
\begin{aligned}
V(t)= & w_{k}^{T}(t) P_{k} w_{k}(t)+\int_{t-\tau(t)}^{t} w_{k}^{T}(s) Q_{k} w_{k}(s) d s \\
& +\int_{t-\tau(t)}^{t} \int_{\theta}^{t} \dot{w}_{k}^{T}(s) S_{k} \dot{w}_{k}(s) d s d \theta .
\end{aligned}
$$

Then $\dot{V}(t)$ can be expressed as

$$
\begin{aligned}
\dot{V}(t)= & 2 w_{k}^{T}(t) P_{k}\left[J(t) w_{k}(t)+c \lambda_{k} A w_{k}(t-\tau(t))\right] \\
& +w_{k}^{T}(t) Q_{k} w_{k}(t) \\
& -(1-\dot{\tau}(t)) w_{k}^{T}(t-\tau(t)) Q_{k} w_{k}(t-\tau(t)) \\
& +\tau(t) \dot{w}_{k}^{T}(t) S_{k} \dot{w}_{k}(t) \\
& -(1-\dot{\tau}(t)) \int_{t-\tau(t)}^{t} \dot{w}_{k}^{T}(s) S_{k} \dot{w}_{k}(s) d s \\
\leq & 2 w_{k}^{T}(t) P_{k}\left[J(t) w_{k}(t)+c \lambda_{k} A w_{k}(t-\tau(t))\right] \\
& +w_{k}^{T}(t) Q_{k} w_{k}(t) \\
& -(1-\mu) w_{k}^{T}(t-\tau(t)) Q_{k} w_{k}(t-\tau(t)) \\
& +\tau \dot{w}_{k}^{T}(t) S_{k} \dot{w}_{k}(t) \\
& -(1-\mu) \int_{t-\tau(t)}^{t} \dot{w}_{k}^{T}(s) S_{k} \dot{w}_{k}(s) d s .
\end{aligned}
$$

From Lemma 6, for any constant matrices $M_{1 k}, M_{2 k}$

$$
\begin{aligned}
& -\int_{t-\tau(t)}^{t} \dot{w}_{k}^{T}(s) S_{k} \dot{w}_{k}(s) d s \\
& \quad \leq \eta_{k}^{T}(t)\left[\begin{array}{cc}
M_{1 k}^{T}+M_{1 k} & -M_{1 k}^{T}+M_{2 k} \\
* & -M_{2 k}^{T}-M_{2 k}
\end{array}\right] \eta_{k}(t) \\
& \quad+\tau \eta_{k}^{T}(t)\left[\begin{array}{c}
M_{1 k}^{T} \\
M_{2 k}^{T}
\end{array}\right] S_{k}^{-1}\left[M_{1 k} M_{2 k}\right] \eta_{k}(t),
\end{aligned}
$$

where

$$
\eta_{k}^{T}(t)=\left[w_{k}^{T}(t), w_{k}^{T}(t-\tau(t))\right] .
$$

Then

$\dot{V}(t)$

$$
\leq \eta_{k}^{T}(t)\left(H_{1 k}+\tau \Gamma_{1}^{T} S_{k} \Gamma_{1}+\tau(1-\mu) \Gamma_{2}^{T} S_{k}^{-1} \Gamma_{2}\right) \eta_{k}(t) .
$$

By Schur complement, $H_{1 k}+\tau \Gamma_{1}^{T} S_{k} \Gamma_{1}+\tau(1-\mu) \Gamma_{2}^{T} S_{k}^{-1} \Gamma_{2}$ is equivalent to expression (13). Then the proof is completed.

In the following criteria, we will introduce Finsler's Lemma. Combining with the Finsler's Lemma, convergent LMI relaxations for synchronization analysis are proposed.

Theorem 8. From Lemma 5, dynamical system (2) is asymptotically synchronized if there exist $P_{k}=P_{k}^{T}>0, Q_{k}=Q_{k}^{T}>$ $0, S_{k}=S_{k}^{T}>0, Y_{k}=Y_{k}^{T}>0$, and any real matrices $M_{i k},(i=1,2)$, and the following LMIs hold for all $2 \leq k \leq N$ :

$$
\left(\mathbb{B}^{\perp}\right)^{T} \Xi_{2 k} \mathbb{B}^{\perp}<0,
$$


where

$$
\begin{aligned}
\mathbb{B} & =\left[J(t), c \lambda_{k} A,-I_{N}\right], \\
\Xi_{2 k} & =\left[\begin{array}{ccc}
H_{2 k} & \tau \Gamma_{1}^{T} Y_{k}^{T} & \tau(1-\mu) \Gamma_{2}^{T} \\
* & -Y_{k}-Y_{k}^{T}+\tau S_{k} & 0 \\
* & * & -\tau(1-\mu) S_{k}
\end{array}\right]<0, \\
\Gamma_{1} & =\left[\begin{array}{ll}
J(t), c \lambda_{k} A
\end{array}\right], \\
\Gamma_{2} & =\left[\begin{array}{ll}
M_{1 k}, M_{2 k}
\end{array}\right], \\
H_{2 k} & =\left[\begin{array}{cc}
\Omega_{11} & \Omega_{12} \\
* & \Omega_{22}
\end{array}\right] \cdot \\
\Omega_{11} & =P_{k} J(t)+J(t)^{T} P_{k}+Q_{k}+(1-\mu)\left(M_{1 k}^{T}+M_{1 k}\right) \\
\Omega_{12} & =P_{k} c \lambda_{k} A-(1-\mu)\left(M_{1 k}^{T}-M_{2 k}\right) \\
\Omega_{22} & =-(1-\mu) Q_{k}-(1-\mu)\left(M_{2 k}^{T}+M_{2 k}\right) .
\end{aligned}
$$

Proof. Choose the same LKF in Theorem 7. From system (9), the following equation holds for any matrices $Y_{k}(2 \leq k \leq N)$,

$$
\begin{aligned}
0= & 2 \dot{w}_{k}^{T}(t) \\
& \cdot Y_{k}\left[-\dot{w}_{k}(t)+J(t) w_{k}(t)+c \lambda_{k} A w_{k}(t-\tau(t))\right] .
\end{aligned}
$$

Combing (16), (17), and (22), we can obtain

$$
\dot{V}_{k}(t) \leq \zeta_{k}^{T}(t) \Xi_{2 k} \zeta_{k}(t),
$$

where

$$
\zeta_{k}^{T}(t)=\left[w_{k}^{T}(t), w_{k}^{T}(t-\tau(t)), \dot{w}_{k}^{T}(t)\right]
$$

Note $\mathbb{B} \zeta_{k}(t)=0$; it follows from Lemma 5 that $\left(\mathbb{B}^{\perp}\right)^{T} \Xi_{2 k} \mathbb{B}^{\perp}<0$ is equivalent to $\zeta_{k}^{T}(t) \Xi_{2 k} \zeta_{k}(t)<0$.

Remark 9. Convergent LMI relaxations are introduced by Finsler's Lemma with homogenous matrix. Then more matrix-valued coefficients can be introduced to reduce conservatism. Moreover, our methods can also be applied to most of the existing synchronization results, such as [6-21].

Remark 10. Different from $[24,25]$ which divide the constant delay part into many more same size delay, the interval $[0, \tau(t)]$ can be chosen arbitrarily into smaller variable subintervals $[0, \rho \tau(t)],[\rho \tau(t), \tau(t)]$, where $\rho \in(0,1)$.

Theorem 11. From Lemma 5, for given scalar $0<\rho<1$, system (2) is synchronized if there exist $P_{k}=P_{k}^{T}>0, Q_{k}=$ $Q_{k}^{T}>0, R_{k}=R_{k}^{T}>0, W_{k}=W_{k}^{T}>0, S_{i k}=S_{k}^{T}>0,(i=1,2)$, and real matrices $Y_{k}, M_{i k}$, and $T_{i k},(i=1,2)$, and the following LMIs hold for all $2 \leq k \leq N$ :

$$
\left(\mathbb{B}^{\perp}\right)^{T} \Xi_{3 k} \mathbb{B}^{\perp}<0,
$$

where

$$
\begin{aligned}
& \mathbb{B}=\left[J(t), c \lambda_{k} A, 0,0,-I_{N}\right], \\
& \Xi_{3 k}=\left[\begin{array}{ccccc}
H_{3 k}^{(1)} & 0 & \tau \Gamma_{1}^{T} Y_{k}^{T} & \tau(1-\rho \mu) \Gamma_{2}^{T} & 0 \\
* & H_{3 k}^{(2)} & 0 & 0 & \tau(1-\rho)(1-\rho \mu) \Gamma_{3}^{T} \\
* & * & H_{3 k}^{(3)} & 0 & 0 \\
* & * & * & -\tau(1-\rho \mu) S_{1 k} & 0 \\
* & * & * & * & -\tau(1-\rho)(1-\rho \mu) S_{2 k}
\end{array}\right]<0, \\
& H_{3 k}^{(1)}=\left[\begin{array}{cc}
\Omega_{11}^{(1)} & \Omega_{12}^{(1)} \\
* & \Omega_{22}^{(1)}
\end{array}\right] \text {. } \\
& \Omega_{11}^{(1)}=P_{k} J(t)+J(t)^{T} P_{k}+Q_{k}+R_{k}+W_{k}+(1-\rho \mu)\left(M_{1 k}^{T}+M_{1 k}\right) \\
& \Omega_{12}^{(1)}=P_{k} c \lambda_{k} A-(1-\rho \mu)\left(M_{1 k}^{T}-M_{2 k}\right) \\
& \Omega_{22}^{(1)}=-(1-\mu) Q_{k}-(1-\mu)\left(M_{2 k}^{T}+M_{2 k}\right) \\
& H_{3 k}^{(2)}=\left[\begin{array}{cc}
\Omega_{11}^{(2)} & \Omega_{12}^{(2)} \\
* & \Omega_{22}^{(2)}
\end{array}\right] . \\
& \Omega_{11}^{(2)}=(1-\rho \mu)\left(T_{1 k}^{T}+T_{1 k}\right)-(1-\rho \mu) R_{k} \\
& \Omega_{12}^{(2)}=-(1-\rho \mu)\left(T_{1 k}^{T}-T_{2 k}\right)
\end{aligned}
$$




$$
\begin{aligned}
\Omega_{22}^{(2)} & =-(1-\rho \mu)\left(T_{2 k}^{T}+T_{2 k}\right)-W_{k} . \\
\Gamma_{1} & =\left[J(t), c \lambda_{k} A\right], \\
\Gamma_{2} & =\left[M_{1 k}, M_{2 k}\right], \\
\Gamma_{3} & =\left[T_{1 k}, T_{2 k}\right] \\
H_{3 k}^{(3)} & =-Y_{k}-Y_{k}^{T}+\rho \tau S_{1 k}+(\tau-\rho \tau) S_{2 k} .
\end{aligned}
$$

Proof. The LKF is confined in the following inequality:

$$
V_{k}(t)=V_{1 k}(t)+V_{2 k}(t)+V_{3 k}(t)+V_{4 k}(t)+V_{5 k}(t),
$$

where

$$
\begin{aligned}
V_{1 k}(t)= & w_{k}^{T}(t) P_{k} w_{k}(t) \\
V_{2 k}(t)= & \int_{t-\tau(t)}^{t} w_{k}^{T}(s) Q_{k} w_{k}(s) d s \\
V_{3 k}(t)= & \int_{t-\rho \tau(t)}^{t} w_{k}^{T}(s) R_{k} w_{k}(s) d s \\
& +\int_{t-\tau}^{t} w_{k}^{T}(s) W_{k} w_{k}(s) d s \\
V_{4 k}(t)= & \int_{t-\rho \tau(t)}^{t} \int_{\theta}^{t} \dot{w}_{k}^{T}(s) S_{1 k} \dot{w}_{k}(s) d s d \theta \\
V_{5 k}(t)= & \int_{t-\tau}^{t-\rho \tau(t)} \int_{\theta}^{t} \dot{w}_{k}^{T}(s) S_{2 k} \dot{w}_{k}(s) d s d \theta .
\end{aligned}
$$

Then $\dot{V}(t)$ can be expressed as

$$
\begin{aligned}
& \dot{V}_{1 k}(t)=2 w_{k}^{T}(t) P_{k}\left[J(t) w_{k}(t)+c \lambda_{k} A w_{k}(t-\tau(t))\right] \\
& \dot{V}_{2 k}(t) \\
& \quad=w_{k}^{T}(t) Q_{k} w_{k}(t) \\
& \quad-(1-\dot{\tau}(t)) w_{k}^{T}(t-\tau(t)) Q_{k} w_{k}(t-\tau(t)) \\
& \dot{V}_{3 k}(t) \\
& \quad=w_{k}^{T}(t) R_{k} w_{k}(t) \\
& \quad-(1-\rho \dot{\tau}(t)) w_{k}^{T}(t-\rho \tau(t)) R_{k} w_{k}(t-\rho \tau(t)) \\
& \quad+w_{k}^{T}(t) W_{k} w_{k}(t)-w_{k}^{T}(t-\tau) W_{k} w_{k}(t-\tau)
\end{aligned}
$$

$\dot{V}_{4 k}(t)$

$$
\begin{aligned}
= & \rho \tau(t) \dot{w}_{k}^{T}(t) S_{1 k} \dot{w}_{k}(t) \\
& -(1-\rho \dot{\tau}(t)) \int_{t-\tau(t)}^{t} \dot{w}_{k}^{T}(s) S_{1 k} \dot{w}_{k}(s) d s
\end{aligned}
$$

$$
\begin{aligned}
\dot{V}_{5 k}(t) & \\
\quad= & (\tau-\rho \tau(t)) \dot{w}_{k}^{T}(t) S_{2 k} \dot{w}_{k}(t) \\
& \quad-(1-\rho \dot{\tau}(t)) \int_{t-\tau(t)}^{t-\rho \tau(t)} \dot{w}_{k}^{T}(s) S_{2 k} \dot{w}_{k}(s) d s .
\end{aligned}
$$

From Lemma 6, for any constant matrices $M_{1 k}, M_{2 k}$

$$
\begin{aligned}
& -\int_{t-\tau(t)}^{t} \dot{w}_{k}^{T}(s) S_{1 k} \dot{w}_{k}(s) d s \\
& \leq \eta_{1 k}^{T}(t)\left[\begin{array}{cc}
M_{1 k}^{T}+M_{1 k} & -M_{1 k}^{T}+M_{2 k} \\
* & -M_{2 k}^{T}-M_{2 k}
\end{array}\right] \eta_{1 k}(t) \\
& \quad+\tau \eta_{1 k}^{T}(t)\left[\begin{array}{c}
M_{1 k}^{T} \\
M_{2 k}^{T}
\end{array}\right] S_{1 k}^{-1}\left[\begin{array}{ll}
M_{1 k} & M_{2 k}
\end{array}\right] \eta_{1 k}(t),
\end{aligned}
$$

where

$$
\eta_{1 k}^{T}(t)=\left[w_{k}^{T}(t), w_{k}^{T}(t-\tau(t))\right] .
$$

For any appropriate dimension matrices $T_{1 k}, T_{2 k}$, we have

$$
\begin{aligned}
& -\int_{t-\tau}^{t-\rho \tau(t)} \dot{w}_{k}^{T}(s) S_{2 k} \dot{w}_{k}(s) d s \\
& \leq \eta_{2 k}^{T}(t)\left[\begin{array}{cc}
T_{1 k}^{T}+T_{1 k} & -T_{1 k}^{T}+T_{2 k} \\
* & -T_{2 k}^{T}-T_{2 k}
\end{array}\right] \eta_{2 k}(t) \\
& \quad+\tau(1-\rho) \eta_{2 k}^{T}(t)\left[\begin{array}{c}
T_{1 k}^{T} \\
T_{2 k}^{T}
\end{array}\right] S_{2 k}^{-1}\left[\begin{array}{ll}
T_{1 k} & \left.T_{2 k}\right] \eta_{2 k}(t),
\end{array}\right.
\end{aligned}
$$

where

$$
\eta_{2 k}^{T}(t)=\left[w_{k}^{T}(t-\rho \tau(t)), w_{k}^{T}(t-\tau)\right] .
$$

From system (9), the following equation holds for any matrices $Y_{k}(2 \leq k \leq N)$ :

$$
\begin{aligned}
0= & 2 \dot{w}_{k}^{T}(t) \\
& \cdot Y_{k}\left[-\dot{w}_{k}(t)+J(t) w_{k}(t)+c \lambda_{k} A w_{k}(t-\tau(t))\right] .
\end{aligned}
$$

Substituting the proposed equalities,

$$
\dot{V}_{k}(t) \leq \Theta_{k}^{T}(t) \Xi_{3 k} \Theta_{k}(t),
$$




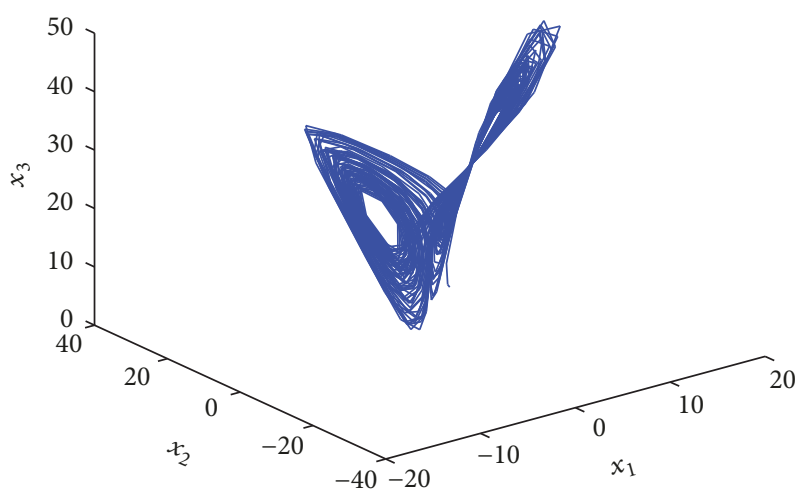

FIgURE 1: The chaotic trajectory of Lorenz systems.

where

$$
\begin{aligned}
& \Theta_{k}^{T}(t)=\left[w_{k}^{T}(t), w_{k}^{T}(t-\tau(t)), w_{k}^{T}(t-\rho \tau(t)),\right. \\
& \left.w_{k}^{T}(t-\tau), \dot{w}_{k}^{T}(t)\right] .
\end{aligned}
$$

From Lemma 5, $\Theta_{k}^{T}(t) \Xi_{3 k} \Theta_{k}(t)<0$ can be acquired. This completes the proof.

Remark 12. Obviously, when we choose different values of $\rho$, the synchronization criteria can also be changed. Through the choice of appropriate parameters $\rho$, different stability results can be obtained.

Remark 13. By using Lemma 6 and introducing Finsler's Lemma, some delay-dependent conditions are acquired in complex networks. The criteria in this paper can be easily used in many existing references and obtain better results, such as [26-30].

\section{Numerical Example}

Consider the Lorenz system in this example

$$
\begin{aligned}
& \dot{x}_{1}=a\left(x_{2}-x_{1}\right) \\
& \dot{x}_{2}=\widehat{c} x_{1}-x_{1} x_{3}-x_{2} \\
& \dot{x}_{3}=x_{1} x_{2}-b x_{3},
\end{aligned}
$$

where $a>0, b>0, \widehat{c}>0$. When $a=10, b=8 / 3, \widehat{c}=28$, the net is chaotic, and its behavior is shown in Figure 1.

We assume $c=1$, and the derivatives of time delays are $\mu=0, \tau=0.2, \Lambda=6 * \operatorname{diag}(1,1,1,1,1)$, and its Jacobian is

$$
\begin{aligned}
J(t) & =\left[\begin{array}{ccc}
-a & a & 0 \\
\widehat{c} & -1 & 0 \\
0 & 0 & -b
\end{array}\right], \\
G & =\left[\begin{array}{cccc}
-3 & 1 & 1 & 1 \\
1 & -3 & 1 & 1 \\
1 & 1 & -3 & 1 \\
1 & 1 & 1 & -3
\end{array}\right],
\end{aligned}
$$

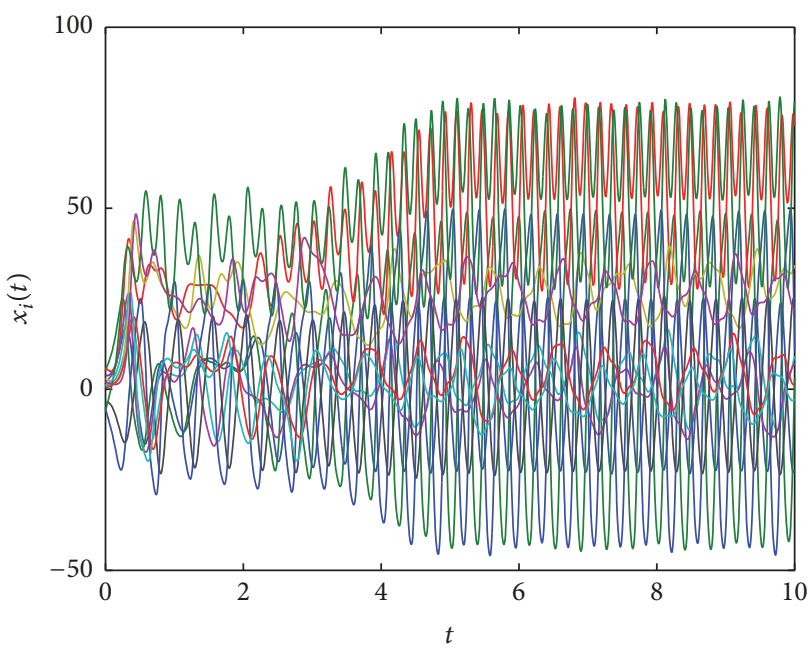

FIGURE 2: States of network (2) without control: $x_{i}(t), i=1,2,3,4$.

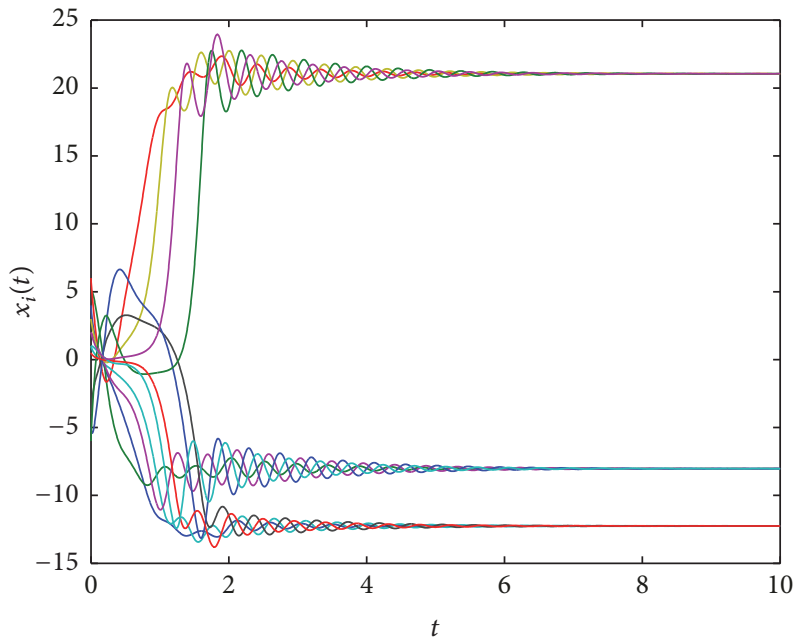

FIGURE 3: States of network (2) with control: $x_{i}(t), i=1,2,3,4$.

$$
A=\left[\begin{array}{ccc}
2.4 & 0 & 2.4 \\
0 & 4.8 & 0 \\
2.4 & 0 & 2.4
\end{array}\right]
$$

Figures 2 and 3 show the states of network (2) without and with control, respectively. Figure 4 shows the synchronization errors between every node, $e_{j}(t)=x_{1 j}(t)-x_{i j}(t)$, for $i=$ $2,3,4, j=1,2,3$. From Figures 3 and 4 , it is easy to see that the system cannot be synchronized by itself, and it can achieve synchronization by pinning control.

\section{Conclusion}

During the past decades, there has been a rapid development of the techniques about complex networks. Numerous studies have shown that complex network is good at dealing with the problem of function approximation and uncertainties. In the paper, a novel analytical method is provided to ensure 

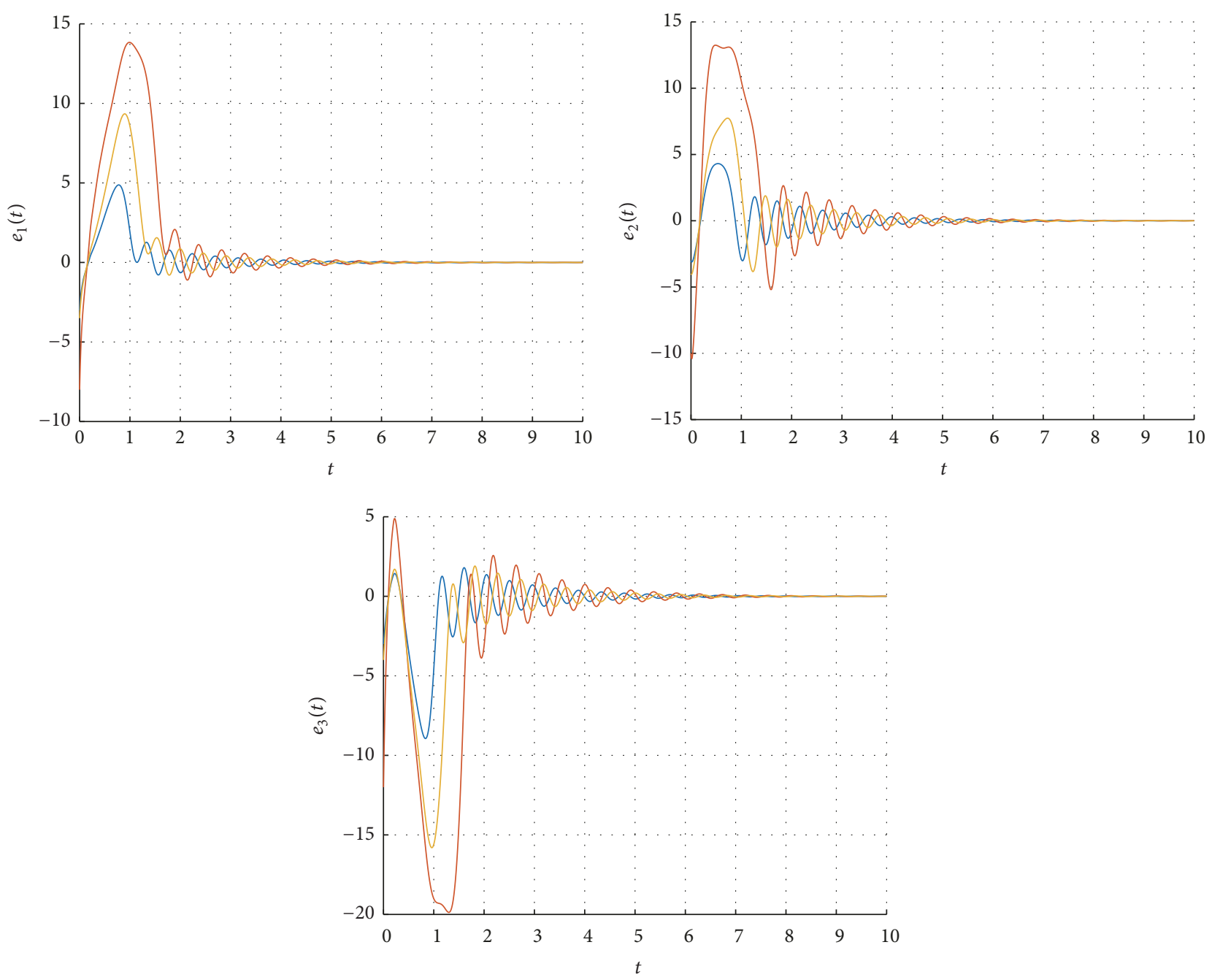

FIGURE 4: Synchronization errors in an example: $e_{j}(t)=x_{1 j}(t)-x_{i j}(t)$ for $i=2,3,4, j=1,2,3$.

the synchronization rigorously for complex system. By using Newton-Leibniz formula and introducing Finsler's Lemma, the obtained synchronization criteria are divided in terms of LMI inequalities, and such method has not been obtained until now. On the other hand, in the studies of network-based motion control in actual models [31-34], a novel integral barrier function is first employed for control design of the constrained distributed parameter system modeled as PDEs [35-38], fault tolerance control in complex systems [39], have been hot topics in recent time. Therefore, how to extend our results into these control fields is still a challenging problem.

\section{Conflicts of Interest}

The authors declare that they have no conflicts of interest.

\section{Acknowledgments}

This work was supported by the National Science Fund for Distinguished Young Scholar (Grant no. 51225503), the
National Natural Science Foundation of China (51305066 and 61603076), and Fundamental Research Funds for the Central Universities (ZYGX2016J116).

\section{References}

[1] H. Zhang, Z. Wang, and D. Liu, "Global asymptotic stability of recurrent neural networks with multiple time-varying delays," IEEE Transactions on Neural Networks, vol. 19, no. 5, pp. 855873, 2008.

[2] Z. Wang, H. Zhang, and B. Jiang, "LMI-based approach for global asymptotic stability analysis of recurrent neural networks with various delays and structures," IEEE Transactions on Neural Networks, vol. 22, no. 7, pp. 1032-1045, 2011.

[3] W. He, Y. Chen, and Z. Yin, "Adaptive neural network control of an uncertain robot with full-state constraints," IEEE Transactions on Cybernetics, vol. 46, no. 3, pp. 620-629, 2016.

[4] W. He, Y. Dong, and C. Sun, "Adaptive neural impedance control of a robotic manipulator with input saturation," IEEE Transactions on Systems, Man, and Cybernetics: Systems, vol. 46, no. 3, pp. 334-344, 2016. 
[5] W. He and Y. Dong, "Adaptive fuzzy neural network control for a constrained robot using impedance learning," IEEE Transactions on Neural Networks and Learning Systems, vol. pp, no. 99, pp. 1-13, 2017.

[6] H. Gao, J. Lam, and G. Chen, "New criteria for synchronization stability of general complex dynamical networks with coupling delays," Physics Letters, Section A: General, Atomic and Solid State Physics, vol. 360, no. 2, pp. 263-273, 2006.

[7] H. Shen, J. H. Park, Z.-G. Wu, and Z. Zhang, "Finite-time $\mathrm{H}_{\infty}$ synchronization for complex networks with semi-Markov jump topology," Communications in Nonlinear Science and Numerical Simulation, vol. 24, no. 1-3, pp. 40-51, 2015.

[8] H. Kang and X. Fu, "Epidemic spreading and global stability of an SIS model with an infective vector on complex networks," Communications in Nonlinear Science and Numerical Simulation, vol. 27, no. 1-3, pp. 30-39, 2015.

[9] L. Zhang, Y. Wang, and Q. Wang, "Synchronization for time-varying complex dynamical networks with differentdimensional nodes and non-dissipative coupling," Communications in Nonlinear Science and Numerical Simulation, vol. 24, no. 1-3, pp. 64-74, 2015.

[10] D. Tong, W. Zhou, X. Zhou, J. Yang, L. Zhang, and Y. Xu, "Exponential synchronization for stochastic neural networks with multi-delayed and Markovian switching via adaptive feedback control," Communications in Nonlinear Science and Numerical Simulation, vol. 29, no. 1-3, pp. 359-371, 2015.

[11] J. Qin, H. Gao, and W. X. Zheng, "Exponential synchronization of complex networks of linear systems and nonlinear oscillators: a unified analysis," IEEE Transactions on Neural Networks and Learning Systems, vol. 26, no. 3, pp. 510-521, 2015.

[12] M. Fang, "Synchronization for complex dynamical networks with time delay and discrete-time information," Applied Mathematics and Computation, vol. 258, no. 1, pp. 1-11, 2015.

[13] Y. Liu, Z. Wang, J. Liang, and X. Liu, "Synchronization and state estimation for discrete-time complex networks with distributed delays," IEEE Transactions on Systems, Man, and Cybernetics B, vol. 38, no. 5, pp. 1314-1325, 2008.

[14] Q. Cheng and J. Cao, "Synchronization of complex dynamical networks with discrete time delays on time scales," Neurocomputing, vol. 151, no. 2, pp. 729-736, 2015.

[15] Z.-J. Bao, G. Wu, and W.-J. Yan, "Control of cascading failures in coupled map lattices based on adaptive predictive pinning control," Journal of Zhejiang University: Science C, vol. 12, no. 10, pp. 828-835, 2011.

[16] H.-Y. Sun, N. Li, D.-P. Zhao, and Q.-L. Zhang, "Synchronization of complex networks with coupling delays via adaptive pinning intermittent control," International Journal of Automation and Computing, vol. 10, no. 4, pp. 312-318, 2013.

[17] C. $\mathrm{Hu}$ and $\mathrm{H}$. Jiang, "Pinning synchronization for directed networks with node balance via adaptive intermittent control," Nonlinear Dynamics, vol. 80, no. 1-2, pp. 295-307, 2015.

[18] L. Zhou, C. Wang, and L. Zhou, "Cluster synchronization on multiple sub-networks of complex networks with nonidentical nodes via pinning control," Nonlinear Dynamics, vol. 83, no. 1-2, pp. 1079-1100, 2016.

[19] S. Li and J. Cao, "Distributed adaptive control of pinning synchronization in complex dynamical networks with nondelayed and delayed coupling," International Journal of Control, Automation and Systems, vol. 13, no. 5, pp. 1076-1085, 2015.

[20] G. Chen, "Pinning control and synchronization on complex dynamical networks," International Journal of Control, Automation and Systems, vol. 12, no. 2, pp. 221-230, 2014.
[21] A. Ghaffari and S. Arebi, "Pinning control for synchronization of nonlinear complex dynamical network with suboptimal SDRE controllers," Nonlinear Dynamics, vol. 83, no. 1-2, pp. 1003-1013, 2016.

[22] C. Li and G. Chen, "Synchronization in general complex dynamical networks with coupling delays," Physica A. Statistical Mechanics and Its Applications, vol. 343, no. 1-4, pp. 263-278, 2004.

[23] X.-M. Zhang, M. Wu, J.-H. She, and Y. He, "Delay-dependent stabilization of linear systems with time-varying state and input delays," Automatica, vol. 41, no. 8, pp. 1405-1412, 2005.

[24] Y. Wang, Z. Wang, and J. Liang, "A delay fractioning approach to global synchronization of delayed complex networks with stochastic disturbances," Physics Letters, Section A: General, Atomic and Solid State Physics, vol. 372, no. 39, pp. 6066-6073, 2008.

[25] Z. Fei, H. Gao, and W. X. Zheng, "New synchronization stability of complex networks with an interval time-varying coupling delay," IEEE Transactions on Circuits and Systems II: Express Briefs, vol. 56, no. 6, pp. 499-503, 2009.

[26] D. Gong, H. Zhang, B. Huang, and Z. Ren, "Synchronization criteria and pinning control for complex networks with multiple delays," Neural Computing and Applications, vol. 22, no. 1, pp. 151-159, 2013.

[27] D. Gong, H. Zhang, Z. Wang, and B. Huang, "Novel synchronization analysis for complex networks with hybrid coupling by handling multitude Kronecker product terms," Neurocomputing, vol. 82, pp. 14-20, 2012.

[28] D. Gong, H. Zhang, Z. Wang, and B. Huang, "Pinning synchronization for a general complex networks with multiple timevarying coupling delays," Neural Processing Letters, vol. 35, no. 3, pp. 221-231, 2012.

[29] D. Gong, F. L. Lewis, L. Wang, and K. Xu, "Synchronization for an array of neural networks with hybrid coupling by a novel pinning control strategy," Neural Networks, vol. 77, pp. 41-50, 2016.

[30] D. Gong, J. Liu, and S. Zhao, "Chaotic synchronisation for coupled neural networks based on T-S fuzzy theory," International Journal of Systems Science, vol. 46, no. 4, pp. 681-689, 2015.

[31] C. Yang, Z. Li, R. Cui, and B. Xu, "Neural network-based motion control of an underactuated wheeled inverted pendulum model," IEEE Transactions on Neural Networks and Learning Systems, vol. 25, no. 11, pp. 2004-2016, 2014.

[32] C. Yang, Y. Jiang, Z. Li, W. He, and C.-Y. Su, "Neural control of bimanual robots with guaranteed global stability and motion precision," IEEE Transactions on Industrial Informatics, In Press.

[33] C. Yang, X. Wang, L. Cheng, and H. Ma, "Neural-learningbased telerobot control with guaranteed performance," IEEE Transactions on Cybernetics, In press.

[34] C. Yang, K. Huang, H. Cheng, Y. Li, and C. Su, "Haptic identification by ELM-controlled uncertain manipulator," IEEE Transactions on Systems, Man, and Cybernetics: Systems, In press.

[35] W. He and S. S. Ge, "Vibration control of a flexible beam with output constraint," IEEE Transactions on Industrial Electronics, vol. 62, no. 8, pp. 5023-5030, 2015.

[36] W. He, C. Sun, and S. S. Ge, "Top tension control of a flexible marine riser by using integral-barrier lyapunov function," IEEE/ASME Transactions on Mechatronics, vol. 20, no. 2, pp. 497-505, 2015. 
[37] W. He and S. S. Ge, "Cooperative control of a nonuniform gantry crane with constrained tension," Automatica, vol. 66, pp. 146-154, 2016.

[38] W. He, Y. Ouyang, and J. Hong, "Vibration control of a flexible robotic manipulator in the presence of input deadzone," IEEE Transactions on Industrial Informatics, vol. 13, no. 1, pp. 48-59, 2017.

[39] X. Wu and D. Gao, "Fault tolerance control of SOFC systems based on nonlinear model predictive control," International Journal of Hydrogen Energy, vol. 42, no. 4, pp. 2288-2308, 2017. 


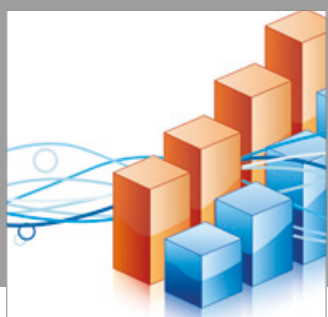

Advances in

Operations Research

vatersals

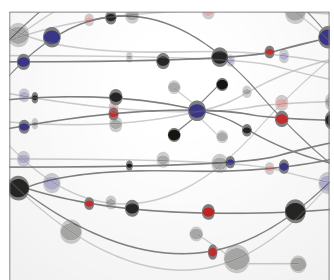

\section{The Scientific} World Journal
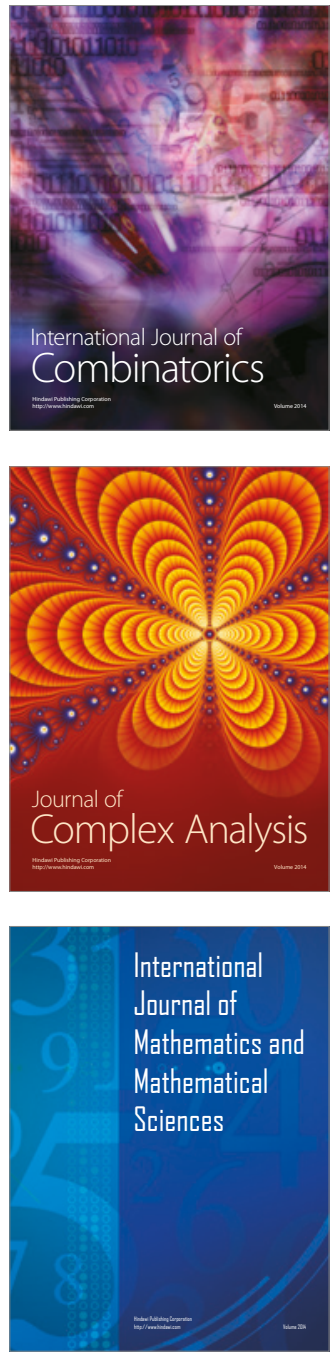
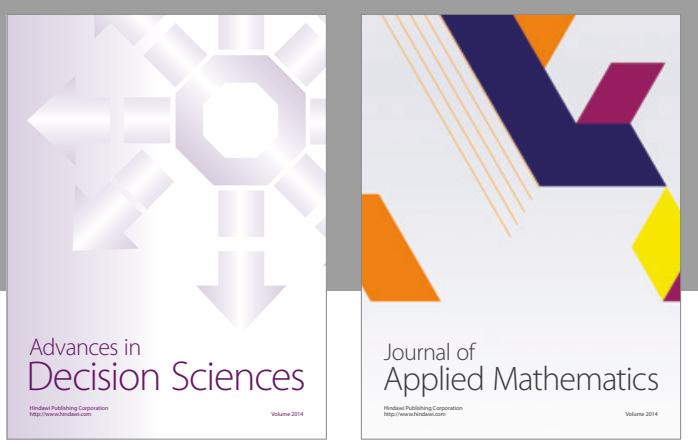

Algebra

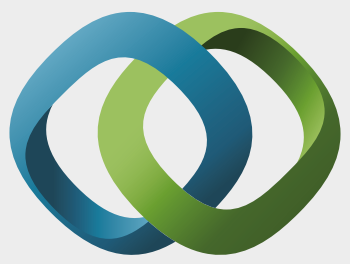

\section{Hindawi}

Submit your manuscripts at

https://www.hindawi.com
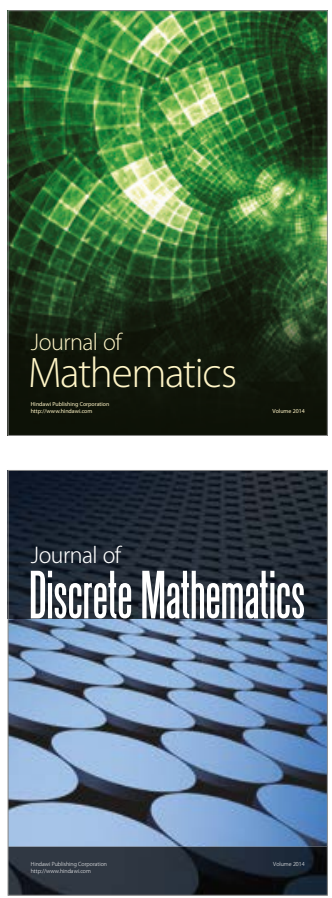

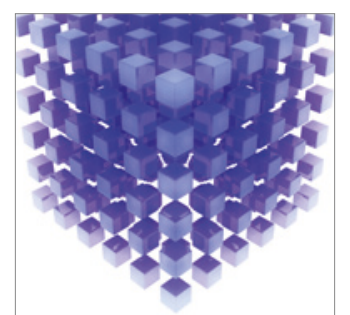

Mathematical Problems in Engineering
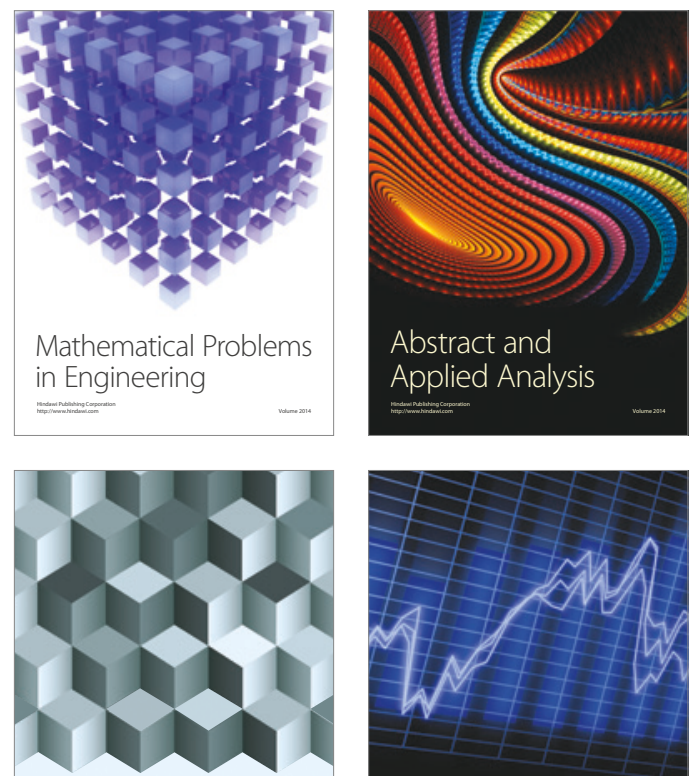

Journal of

Function Spaces

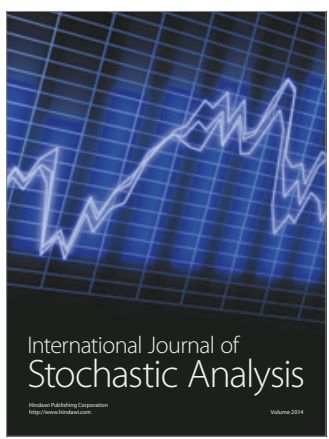

Probability and Statistics
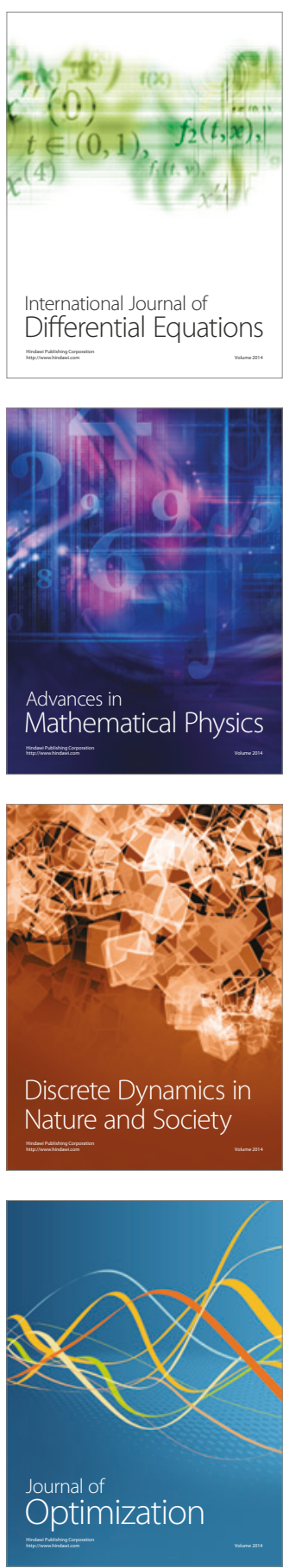